psychopraxis. neuropraxis $2022 \cdot 25: 107-112$ https://doi.org/10.1007/s00739-022-00779-8 Angenommen: 10. Januar 2022 Online publiziert: 31. Januar 2022 ○ Der/die Autor(en) 2022

\section{Q}

\section{Barbara Graf' • Patrick Altmann ${ }^{2}$. Henriette Löffler-Stastka ${ }^{3}$}

'Textil - freie und konzeptuelle künstlerische Praxis und Materialkultur, Universität für angewandte Kunst, Wien, Österreich

${ }^{2}$ Universitätsklinik für Neurologie, Medizinische Universität Wien, Wien, Österreich

${ }^{3}$ Univ.-Klinik für Psychoanalyse und Psychotherapie, Medizinische Universität Wien, Wien, Österreich

\title{
Die subjektive Verarbeitung chronischer Symptome und Schmerz bei Menschen mit Multipler Sklerose: Visualisierung und Externalisierung als Ausdruck der Lebensqualität
}

\section{Einleitung \\ Bewältigungsstrategien bei chronischen Erkrankungen}

Eine kürzlich durchgeführte Metaanalyse hat gezeigt, dass psychische Gesundheit die Lebensqualität und den Outcome bei verschiedenen chronischen Erkrankungen beeinflusst [1]. Veränderbare interne und externe Faktoren werden oft unzureichend behandelt und dargestellt. Dennoch bestimmen diese wiederum, zusammen mit den körperlichen chronischen Bedingungszwängen, Freiheitsgrade für ein selbstbestimmtes Dasein. Lane et al. postulieren, dass eine dauerhafte Veränderung durch die Rekonsolidierung von Erinnerungen erreicht wird [2]. Durch die Aktivierung einer Erinnerung mit dem ursprünglich verbundenen Affekt werden neue emotionale Erfahrungen ermöglicht. Die Anpassung von Sichtweisen erfolgt dabei über neue Erfahrungsrahmen und Wissenskonzepte. Diese können die Wahrnehmung und das Verständnis dahin gehend ändern, dass sie, von intrinsischer und extrinsischer Motivation getrieben, den Affekt eines Einzelnen prägen.

Darüber hinaus hängt die Verfügbarkeit von Bewältigungsstrategien von verleiblichtem Wissen und unbewusstem
Lernen ab. Ziel dieser Arbeit ist, die Interdependenz zwischen der Krankheit (objektive Befunde) und dem Krankheitserleben (subjektive Wahrnehmungsprozesse) aufzuzeigen, um Denkanstöße zur Krankheitsbewältigung und Krankheitsverarbeitung zu ermöglichen. Multiple Sklerose (MS) wird hierfür als Paradigma einer chronischen Erkrankung dargestellt, da sie die häufigste Ursache einer neurologischen Behinderung ausmacht.

\section{Multiple Sklerose als Paradigma neurologischer Beeinträchtigung}

Etwa 2,5 Mio. Menschen leben weltweit mit der Diagnose einer MS, der Altersgipfel bei Diagnosestellung liegt bei 20 bis 40 Jahren, also oft in einer Phase, die vom Erreichen persönlicher Lebensziele geprägt ist, wie etwa einer Ausbildung, Karriere oder Familienplanung [3, 4]. Gegenüber und auch in diesem Kontinuum befinden sich behandelnde Ärztinnen und Ärzte, deren Aufgabe es ist, den Gesundheitszustand ihrer Patientinnen und $\mathrm{Pa}$ tienten zu überwachen. Dies erfolgt bei MS typischerweise über sogenannte „clinician reported outcomes" (CROs), also Therapieziele, wie sie etwa auch in klinischen Zulassungsstudien angewandt werden. Diese umfassen hauptsächlich die klinisch neurologische Symptomatik (clinical disability oder clinical disease activity) oder radiologische Krankheitsaktivität (disease activity on magnetic resonance imaging, auch MRI activity). Darüber hinaus nehmen Biomarker eine wichtige Position ein $[5,6]$. Auf der anderen Seite nehmen „patient reported out-

\footnotetext{
Infobox Informationen über die Abbildungen und Künstlerin

- Graf, Barbara, Stitches and Sutures. In: Envelope \#3, Universität für angewandte Kunst Wien, Zentrum Fokus Forschung. https://publiccolloquium.uni-ak.ac.at/ 2020/ZFF_ArtResearchEnvelope_3_web. pdf. [abgerufen am 16.12.2021]

- Graf, Barbara, Stitches and Sutures: From Physical Sensations to Forms of Perception, Imagination and Representation. In: Envelope \#4, Universität für angewandte Kunst Wien, Zentrum Fokus Forschung Open access in Research Catalogue, 2021. https://www.researchcatalogue.net/ view/1193650/1264769. [abgerufen am 16.12.2021]

- Barbara Graf ist visuelle Künstlerin und Lehrende an der Universität für angewandte Kunst Wien, Abteilung Textil. Seit 2018 Arbeit an Stitches and Sutures als PhD-Projekt künstlerischer Forschung Universität für angewandte Kunst Wien, Zentrum Fokus Forschung; Betreuerin: Barbara Putz-Plecko.

- Abbildungen $\odot$ Barbara Graf
} 


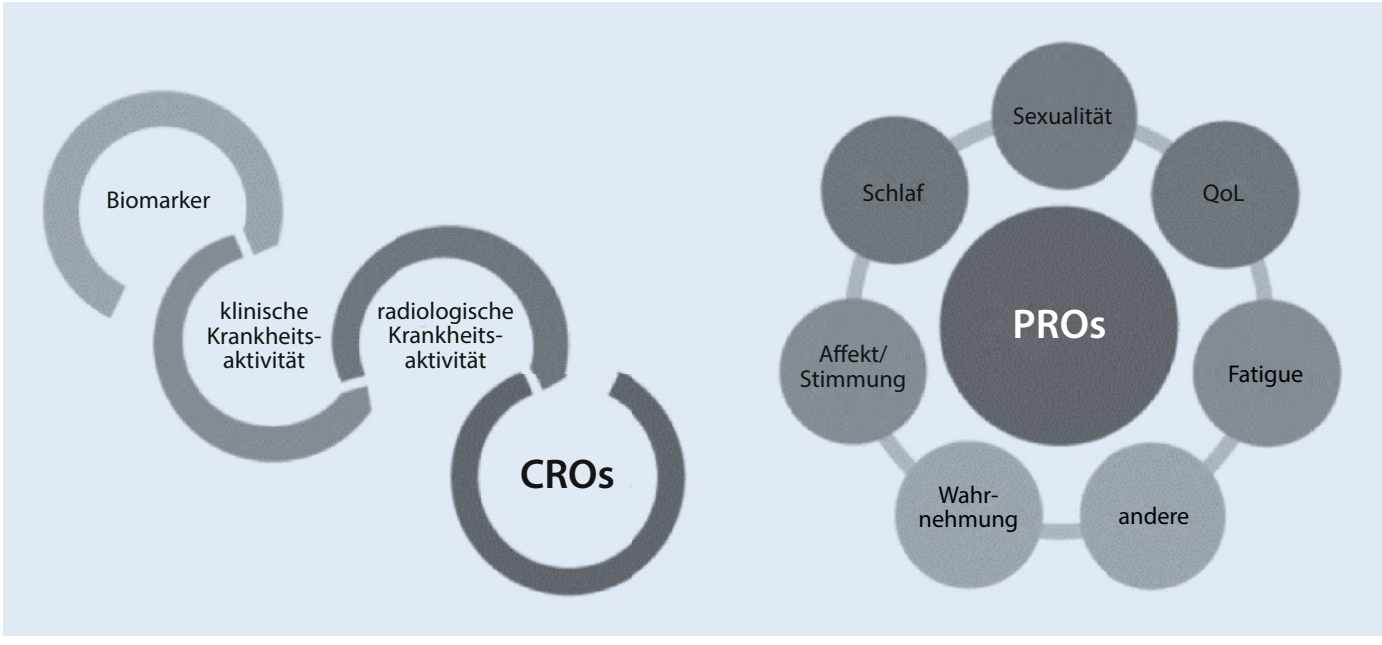

Abb. $1<$ Die Gegenüberstellung von "clinician reported outcomes" (CROS) als klinische Kriterien für die Beurteilung der Krankheitsaktivität bei multipler Sklerose auf der einen Seite und "patient reported outcomes" (PROS) als PatientInnenberichte über den individuellen Gesundheitszustand auf der anderen. QoL quality of life, Lebensqualität (Adaptiert nach Nowinski, Miller und Cella [7])

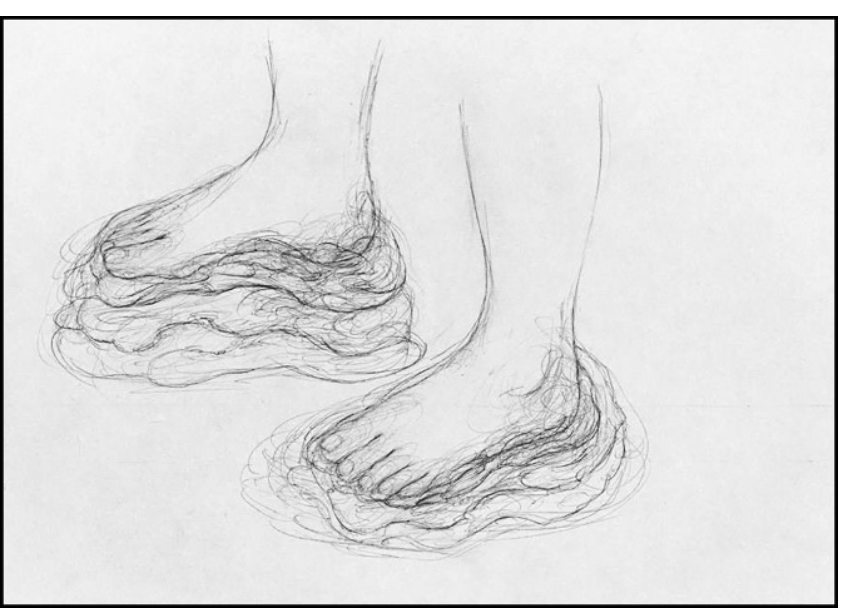

Abb. 2 A Barbara Graf, Zeichnung Nr. 215, 2019, Bleistift auf Papier, $29,7 \times 42 \mathrm{~cm}$. Die Empfindung faserartiger Schichten in verschiedener Höhe unter den Füßen und damit verwachsen

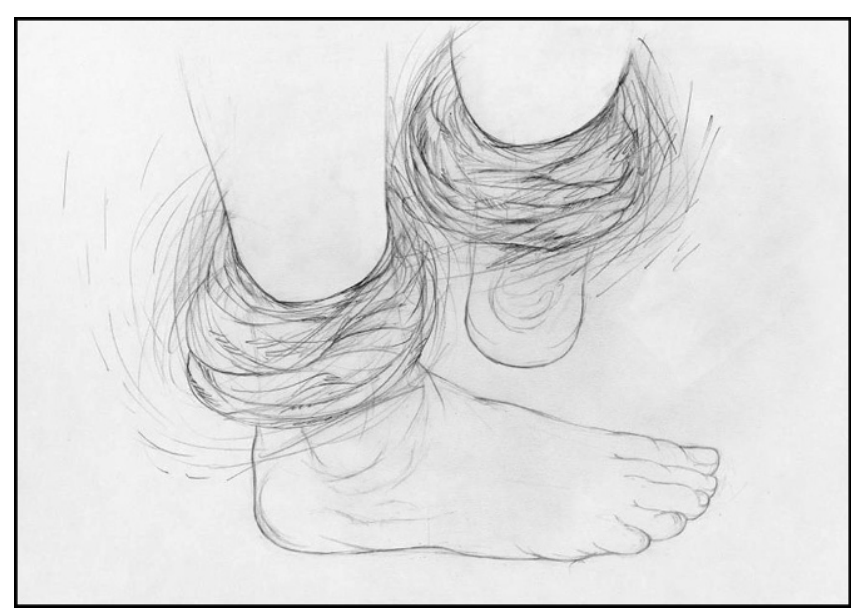

Abb. 3 А Barbara Graf, Zeichnung Nr. 189, 2017, Bleistift auf Papier, $29,7 \times 42 \mathrm{~cm}$. Empfindung eng umwickelter Bandagen comes“ (PROs) stetig an Bedeutung zu. Bei PROs handelt es sich um Berichte von PatientInnen über ihren Gesundheitszustand; sie umfassen verschiedene Lebensbereiche, die auch als gesundheitsbezogene Lebensqualität („,health related quality of life " [HRQoL]) zusammengefasst werden [5]. Die PROs sind vielfältig und dazu gehören etwa, um einige zu nennen, das subjektive Krankheitsempfinden, die erkrankungsbezogene physische Behinderung, Sexualität, Fatigue, affektive Erkrankungen, Schlafqualität u.v.m. Über die Hierarchie dieser Systeme herrscht derzeit kein Konsens (• Abb. 1, adaptiert nach [7]). Das bedeutet, dass derzeit PROs oder die HRQoL im Gegensatz zu CROs keinen evidenzbasierten Einfluss auf Therapieentscheidungen nehmen [8].

\section{Fallbericht}

\section{Krankheit und Krankheitserleben eines Menschen mit MS}

Durch den Kontakt der Füße mit dem Boden treten wir auf und bewegen uns fort. Eine ernsthafte Diagnose kann den Boden unter den Füßen wegziehen; im Fall von MS unter Umständen in einem doppelten Sinne. Störungen in den Füßen und beim Gehen sind häufige Phänomene und die Ungewissheit des Verlaufs der Erkrankung ist verunsichernd (• Abb. 2). In der künstlerischen Untersuchung des durch MS veränderten Körperempfindens gehe ich vom eigenen physischen Erleben aus. Das Projekt ist als zeichnerische Phänomenologie zu verstehen und gleichsam als Verarbeitungsprozess der chronischen Erkrankung. Die Diagnose MS im Jahr 2017 löste ein ambivalentes Gefühl aus. So war es einerseits eine Erleichterung, die auch schon früher erlebten Symptome zuordnen zu können, andererseits wurden dadurch Ängste hervorgerufen, wie ich mit MS leben werde.

\section{Physische Symptome bei MS}

Körperliche Symptome bei MS können belastend oder auch einschränkend sein. Mit körperlichen Veränderungen zu leben, ohne die Symptome konstant mit Ängsten zu verbinden, verlangt nach Verarbeitung. Beim milden Verlauf meiner Erkrankung stehen unangenehme bis schmerzhafte Missempfindungen im Vordergrund, welche mich in der Mobilität kaum einschränken, aber zeitweise 
eine erhebliche Belastung darstellen. Die zeichnerische Untersuchung der physischen Phänomene ermöglicht es mir, den fremd gewordenen Körper wieder als eigenen wahrzunehmen. Störende und schmerzhafte Empfindungen haben durch die künstlerische Visualisierung auch noch einen anderen Ort als denjenigen, an den sie zwangsläufig gebunden sind. Es ist nicht nur ein anderer Ort - der des Papiers -, sondern auch eine andere Qualität durch die Transformation in eine visuelle Darstellung.

》) Körperwahrnehmungen sind subjektiv und benötigen eine Sprache oder Darstellungsform zur Vermittlung

Die zeichnerischen Strukturen stellen zwar physische Empfindungen dar, verkörpern aber durch die Externalisierung gleichermaßen einen psychischen Raum. Der Vorgang des Zeichnens des Gespürten, aber dennoch Unfassbaren, ist ein Prozess des körperlichen Verstehens, um ein Körperselbstverständnis wiederzuerlangen, das sich durch die Störungen nicht als Fragmentiertes versteht.

\section{Die Darstellung von Körperempfindungen}

Sie reichen von irritierend, unangenehm oder belastend, bis hin $\mathrm{zu}$ schmerzhaft und betreffen Phänomene wie Kribbeln, Brennen, Stechen, Taubheit, Dumpfheit, Spannungen und Spastik. Manche verhalten sich wie ein konstantes Hintergrundgeräusch; andere wiederum drängen sich in den Vordergrund und können kurz oder lang anhaltend, intensiver oder schwächer sein. Die visuellen Darstellungen der Symptome dienen der Selbstvergewisserung der seltsamen Phänomene, können aber auch anderen Personen eine Vorstellung des Unsichtbaren geben. Körperwahrnehmungen sind zutiefst subjektiv und benötigen eine Sprache oder Darstellungsform zur Vermittlung. Dies umso mehr, da die Parästhesien nicht auf einem externen Stimulus basieren. Bei manchen Symptomen verhält es sich so, als wären sie von einem Materi- psychopraxis. neuropraxis $2022 \cdot 25$ :

107-112

https://doi.org/10.1007/s00739-022-00779-8

(c) Der/die Autor(en) 2022

B. Graf · P. Altmann · H. Löffler-Stastka

Die subjektive Verarbeitung chronischer Symptome und Schmerz bei Menschen mit Multipler Sklerose:

Visualisierung und

Externalisierung als Ausdruck der Lebensqualität

\section{Zusammenfassung}

Somatosensible Symptome und Schmerz zählen zu jenen Beschwerden chronischer Erkrankungen, die sich - aus ärztlicher Sicht - oft einer objektivierbaren Beurteilung entziehen. Persönlichen Externalisierungsprozessen kann hierbei eine besondere Bedeutung zukommen.

\section{Schlüisselwörter}

Erste-Person-Perspektive - Dritte-PersonPerspektive - Subjektivität - Mixed methods . Verarbeitungsprozesse

\section{Subjective Processing of Chronic Symptoms and Pain in People with Multiple Sclerosis: Visualization and Externalization as an Expression of Quality of Life}

\section{Abstract}

Somatosensitive symptoms and pain are among those complaints of chronic diseases that-from a physician's point of view-often elude objective assessment. Personal externalization processes can be of particular importance here.

\section{Keywords}

First person perspective $\cdot$ Third person perspective · Subjectivity · Mixed methods Resilience processes

al ausgelöste Berührungswahrnehmungen oder als wäre eine Körperstelle mit einer textilen Struktur ergänzt oder ausgestopft. Da ich als visuelle Künstlerin häufig mit Textilien arbeite, vermutete ich zuerst, dass sich die künstlerischen Erfahrungen mit meinen aktuellen physischen Empfindungen verbinden. Andere an MS erkrankte Personen schildern jedoch ihre Phänomene auch häufig als „textile Empfindungen“, wie etwa auf Watte ge-
Hier steht eine Anzeige.

Springer 


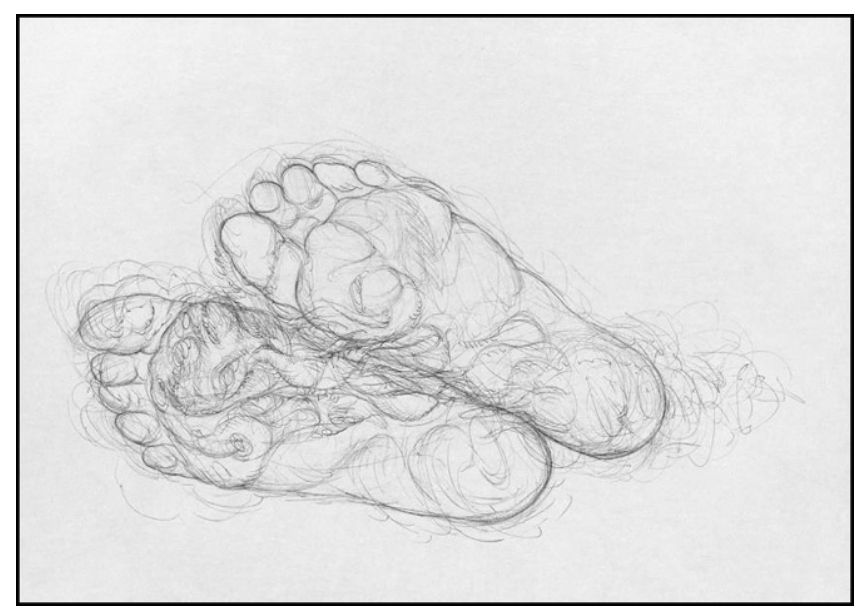

Abb. 4 A Barbara Graf, Zeichnung Nr. 196, 2018, Bleistift auf Papier, $29,7 \times 42 \mathrm{~cm}$. Empfindung mit Fasern durchwachsener Füße

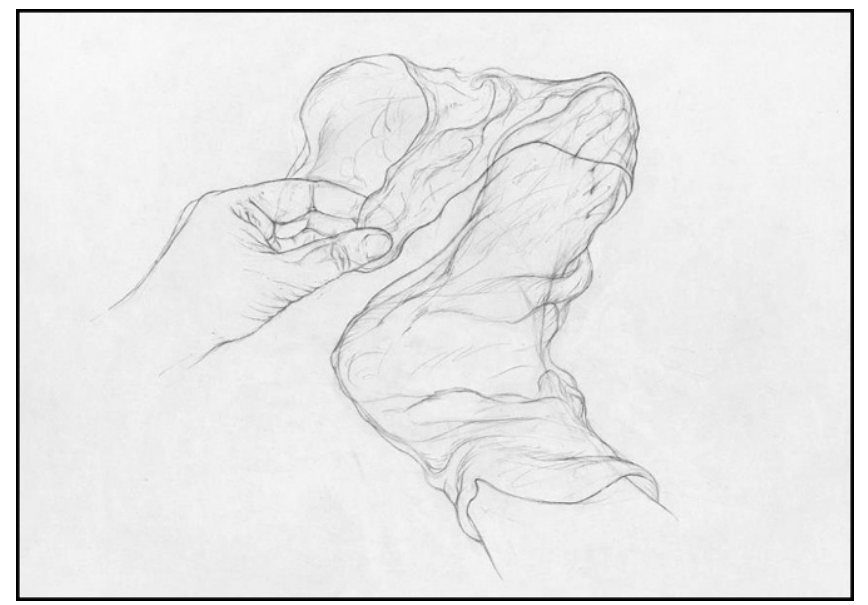

Abb. 6 ム Barbara Graf, Zeichnung Nr. 193, 2018, Bleistift auf Papier, $29,7 \times 42 \mathrm{~cm}$. Empfindung mehrerer Schichten und in Falten gelegter hüllenartiger Fußbekleidungen

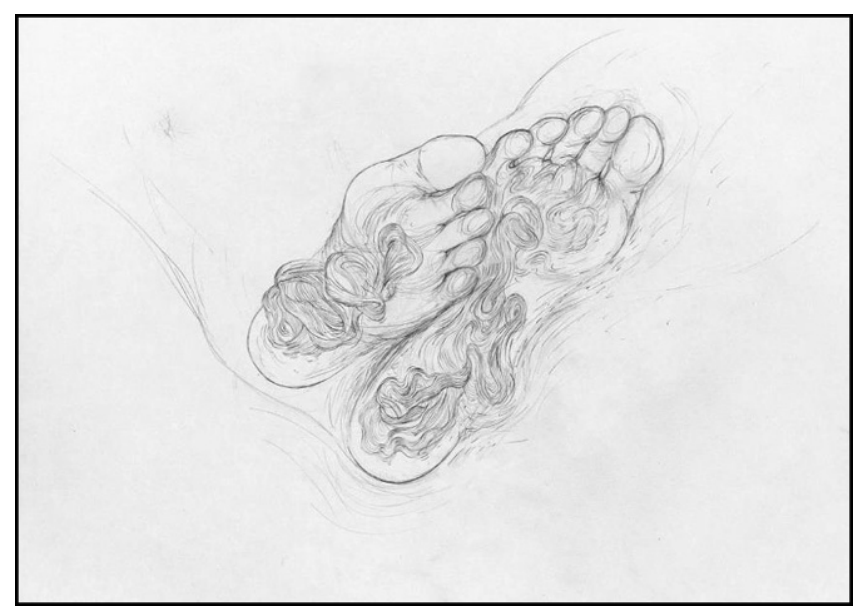

Abb. $5 \Delta$ Barbara Graf, Zeichnung Nr. 202, 2019, Bleistift auf Papier, $29,7 \times 42 \mathrm{~cm}$. Empfindung verschlungener und verknoteter Faserbündel

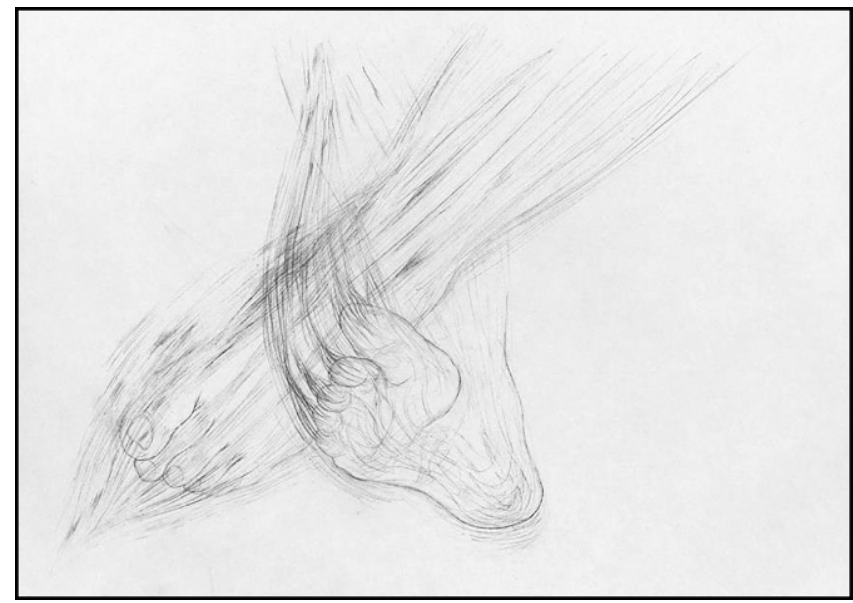

Abb. 7 ॥ Barbara Graf, Zeichnung Nr. 203, 2019, Bleistift auf Papier, $29,7 \times 42 \mathrm{~cm}$. Schmerzhaftes und krampfartiges Ziehen hen, als Socken- oder Handschuhempfindung oder die Empfindung mit engen Bandagen (• Abb. 3) umwickelt zu sein. Auch wenn eine Empfindung als Erlebtes visuell eigentlich nicht darstellbar ist, kann sie sich vermitteln durch das Ereignis, das sie hervorruft, auch wenn es sich um einen nicht vorhandenen externen Stimulus handelt. Die Empfindung der mit Fasern durchwachsenen Fußsohlen (• Abb. 4) kann sich einmal anfühlen als Durchdringung von textilem und körperlichem Gewebe oder ein andermal als verknotete Faserbündel (• Abb. 5), die verwachsen in die Fußsohle über die Körpergrenze hinaus wahrgenommen werden.

Faserartige Dumpfheits- und Taubheitsgefühle (• Abb. 2) sind belastend und die Empfindung knäuelartiger Schichten unter den Füßen verunsichert das Gehen, da sich etwas Illusionäres, aber dennoch Empfundenes zwischen den Boden und die Füße schiebt.

\section{》) Faserartige Dumpfheits- und Taubheitsgefühle sind belastend}

Sockenartige Hüllenempfindungen (• Abb. 6) können bisweilen eine sich selbst vergewissernde Geste auslösen, um zu überprüfen, ob ein Socken getragen wird oder nicht. Ähnlich verhält es sich mit der Empfindung, als würden ausgeleierte Socken unter das Fußgewölbe rutschen. Es ist bisweilen nicht zu unterscheiden, ob es sich um eine materielle
Berührungs- und Druckwahrnehmung handelt oder etwa eine Nervenstörung, die sich mit einer Alltagserfahrung verbindet.

Spannungsempfindungen haben häufig den Charakter, über die Körpergrenze hinaus wahrgenommen $\mathrm{zu}$ werden (- Abb. 7). Ein anderes Mal sind es Spannungen kombiniert mit Faserakkumulationen, die sich von Knäueln zwischen den Zehen als brennendes Ziehen in die Umgebung fortsetzen (• Abb. 8).

Statt die physischen Empfindungen über den vermeintlichen Stimulus zu zeichnen, isoliere ich in einer anderen Darstellungsweise die Strukturen von der Körperstelle. Die in fünf Feldern dargestellten Strukturen sind angelehnt an die zehnstufige Schmerzskala und 


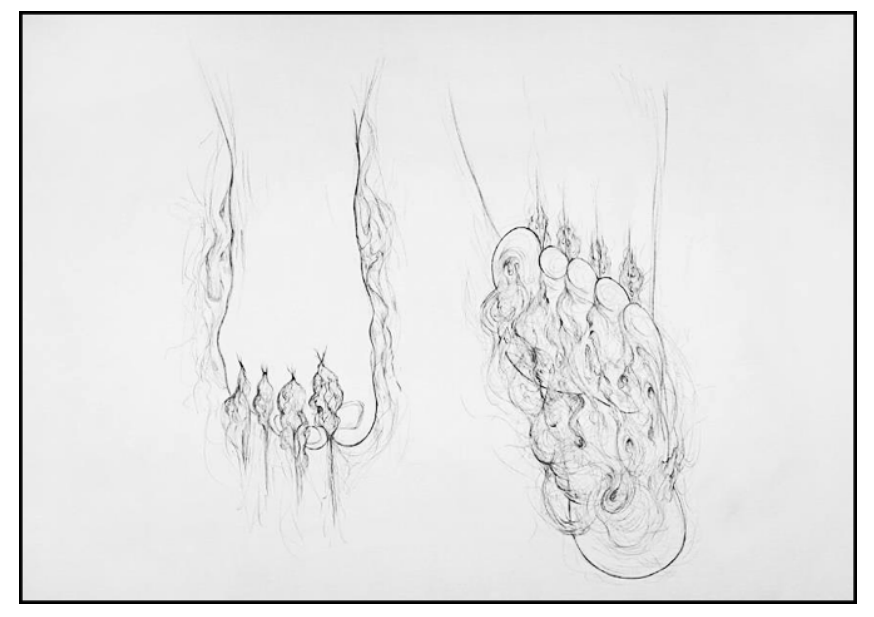

Abb. $8<$ Barbara Graf, Zeichnung Nr. 250, 2021, Bleistift auf Papier, $29,7 \times 42 \mathrm{~cm}$. Faserartige Knäuel unter den Füßen, zwischen den Zehen und brennende Spannung

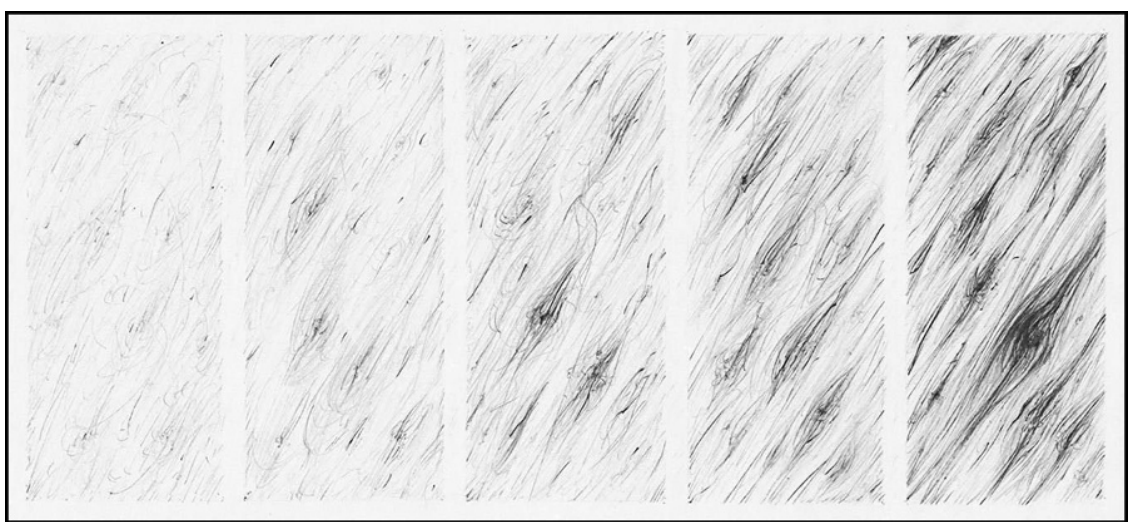

Abb. 9 ॥ Barbara Graf, Zeichnung Nr. 227, 2020, Bleistift auf Papier, 11,7×27 cm, 2020. Ziehende und faserartig stechende Symptome: von irritierender Intensität bis zur Schmerzempfindung

zeigen die Symptome von der Intensität des gerade Wahrnehmbaren bis hin zur schmerzhaften Intensität (• Abb. 9).

Die Verlagerung störender und schmerzhafter Empfindungen in die Bleistiftlinie und auf das Papier ermöglicht durch diesen Transfer, die Symptome anders zu betrachten. Die Missempfindungen werden nicht nur passiv hingenommen, sondern durch die zeichnerische Tätigkeit quasi in die eigene Hand genommen, und der Prozess der Introspektion bis hin zur zeichnerischen Externalisierung bedeutet eine aktive Beschäftigung. Auch wenn die physischen Phänomene durch die externalisierte und künstlerische Existenz nicht verschwinden, verändert sich durch die neue Betrachtungsweise ihre Position und sie zeigen sich auch noch von einer anderen Seite als nur von einer belastenden.

\section{Konklusion}

Eine Motivation und die Fähigkeit zur Einflussnahme können dazu beitragen, die Selbstwirksamkeit und Lebensqualität von Menschen mit chronischen Erkrankungen zu kräftigen. Insbesondere bei der Betreuung von Menschen mit multipler Sklerose treffen unterschiedliche Sichtweisen in einer therapeutischen Beziehung aufeinander. Im Idealfall bieten diese einen gemeinsamen Raum, um komplementär aufeinander einzuwirken. Denn so können physische Phänomene zwar nicht verschwinden, aber die Sichtweise kann eine neue Position ermöglichen - und im Idealfall eine belastende durch eine bewältigende Sicht getauscht werden.

\section{Fazit für die Praxis}

- In der Betreuung von Menschen mit chronischen Erkrankungen wirken sogenannte "clinician reported outcomes" (CROs) und "patient reported outcomes" (PROs). PROs sind Berichte von Patientinnen und Patienten über ihren Gesundheitszustand, ohne dass sie vom behandelnden Arzt oder der behandelnden Ärztin beeinflusst werden. Sie geben Aufschluss über die gesundheitsbezogene Lebensqualität.

- Persönliche Externalisierungsprozesse können helfen, Schmerzen zu bewältigen. Dabei werden unerwünschte und unerträgliche Regungen an das Außen zugeschrieben, wobei der projizierte Impuls dann bei der projizierenden Person unbewusst/verdrängt bleibt, womit veränderte Sichtweisen ermöglicht werden können.

- Insbesondere das somatosensible Nervensystem betreffende Beschwerden entziehen sich oft einer objektivierbaren Messbarkeit. Der individuellen Sichtweise Betroffener einen Raum zu geben, kommt dabei eine besondere Bedeutung zu.

- Dieser Artikel beschreibt den Bezug einer von MS betroffenen Frau, somatosensible Symptome und Schmerz als Beispiel für einen Externalisierungsprozess zu visualisieren:

- Veränderte Körperwahrnehmungen

- Textile Empfindungen

- Mit Fasern durchwachsene Fußsohlen

- Faserartige Dumpfheits- und Taubheitsgefühle

- Sockenartige Hüllenempfindungen

- Spannungsempfindungen

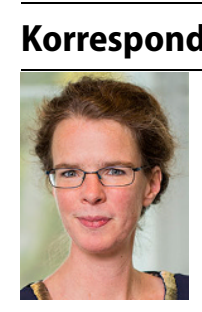

Univ.-Prof. PD Dr.

Henriette Löffler-Stastka

Univ.-Klinik für Psychoanalyse und Psychotherapie,

Medizinische Universität

Wien

Währinger Gürtel 18-20, 1090 Wien, Österreich henriette.loeffler-stastka@ meduniwien.ac.at 


\section{Psychiatrie}

Funding. Open access funding provided by Medical University of Vienna.

\section{Einhaltung ethischer Richtlinien}

Interessenkonflikt. B. Graf, P. Altmann und H. LöfflerStastka geben an, dass kein Interessenkonflikt besteht

Für diesen Beitrag wurden von den Autoren keine Studien an Menschen oder Tieren durchgeführt. Für die aufgeführten Studien gelten die jeweils dort angegebenen ethischen Richtlinien.

Open Access. Dieser Artikel wird unter der Creative Commons Namensnennung 4.0 International Lizenz veröffentlicht, welche die Nutzung, Vervielfältigung Bearbeitung, Verbreitung und Wiedergabe in jeglichem Medium und Format erlaubt, sofern Sie den/die ursprünglichen Autor(en) und die Quelle ordnungsgemäß nennen, einen Link zur Creative Commons Lizenz beifügen und angeben, ob Änderungen vorgenommen wurden.

Die in diesem Artikel enthaltenen Bilder und sonstiges Drittmaterial unterliegen ebenfalls der genannten Creative Commons Lizenz, sofern sich aus der Abbildungslegende nichts anderes ergibt. Sofern das betreffende Material nicht unter der genannten Creative Commons Lizenz steht und die betreffende Handlung nicht nach gesetzlichen Vorschriften erlaubt ist, ist für die oben aufgeführten Weiterverwendungen des Materials die Einwilligung des jeweiligen Rechteinhabers einzuholen.
Weitere Details zur Lizenz entnehmen Sie bitte de Lizenzinformation auf http://creativecommons.org/ licenses/by/4.0/deed.de.

\section{Literatur}

1. Daré LO, Bruand P-E, Gérard D, Marin B, Lameyre V Boumédiène F, Preux PM (2019) Co-morbidities of mental disorders and chronic physical diseases in developing and emerging countries: a metaanalysis. BMC Public Health 19(1):304. https://doi. org/10.1186/s12889-019-6623-6

2. Lane RD, Ryan L, Nadel L, Greenberg L (2015) Memory reconsolidation, emotional arousal, and the process of change in psychotherapy: New insights from brain science. Behav Brain Sci 38:e1. https://doi.org/10.1017/S0140525X14000041

3. Dobson R, Giovannoni G (2019) Multiple sclerosis-a review. Eur J Neurol 26:27-40. https://doi. org/10.1111/ene.13819

4. Kobelt G, Thompson A, Berg J, Gannedahl M, Eriksson J (2017) New insights into the burden and costs of multiple sclerosis in Europe. Mult Scler 23:1123-1136. https://doi.org/10.1177/ 1352458517694432

5. Oh J, Vidal-Jordana A, Montalban X (2018) Multiple sclerosis: clinical aspects. Curr Opin Neurol 31:752-759. https://doi.org/10.1097/wco. 0000000000000622

6. Altmann P et al (2020) Serum neurofilament light chain withstands delayed freezing and repeated thawing. Sci Rep 10:19982

7. Nowinski CJ, Miller DM, Cella D (2017) Evolution of patient-reported outcomes and their role in multiple sclerosis clinical trials. Neurotherapeutics 14:934-944. https://doi.org/10.1007/s13311 017-0571-6

8. Altmann P et al (2021) The smartphone app haMSterfor tracking patient-reported outcomes in people with multiple sclerosis: protocol for a pilot study. JMIR Res Protoc 10:e25011. https://doi.org/ $10.2196 / 25011$

Hinweis des Verlags. Der Verlag bleibt in Hinblick auf geografische Zuordnungen und Gebietsbezeichnungen in veröffentlichten Karten und Institutsadressen neutral.

Hier steht eine Anzeige.

\section{Springer}

